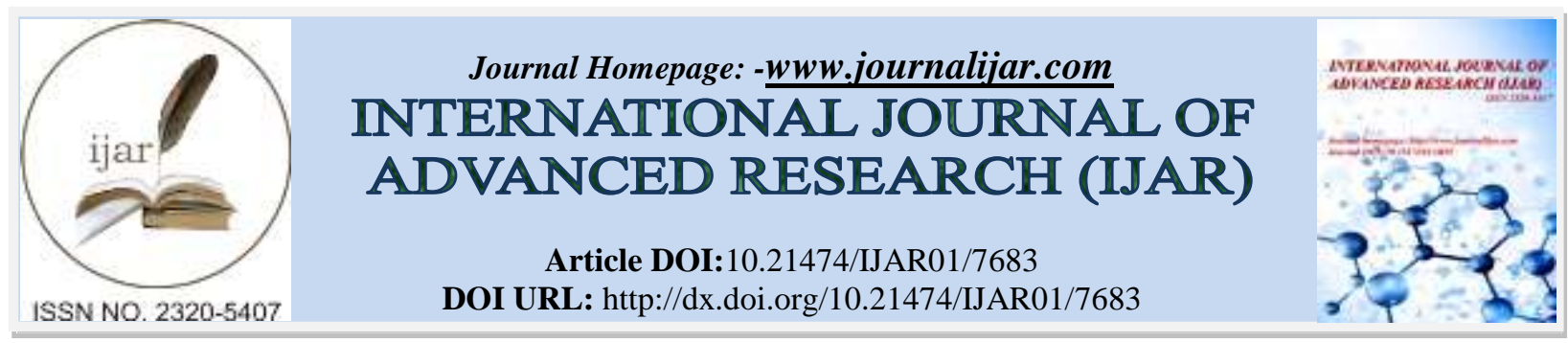

RESEARCH ARTICLE

\title{
ACUTE HYDROCEPHALUS SECONDARY TO INTRAVENTRICULAR NEUROCYSTICERCOSIS CAUSING SUDDEN DEATH.
}

Dr. Agrata Sharma ${ }^{1}$ and Dr. Amit Raj ${ }^{2}$.

1. Senior Resident, UCMS \& GTB HOSPITAL Delhi- 95.

2. Senior Resident, AIIMS New Delhi-29.

\section{Manuscript Info}

Manuscript History

Received: 18 July 2018

Final Accepted: 24 August 2018

Published: September 2018

Keywords:-

Intraventricular, Neurocysticercosis,

Helminthic, Infection, cephalgia.

\section{Abstract}

Neucysticercosis (NCC) is the most common Helminthic infection of the central nervous system and its epidemiology is changing due to increasing travel and migration. Evidence to guide management of the intraventricular form is limited. The intraventricularvariant of NCC is less common than parenchymal disease and usually presents with acutely raised intracranial pressure and untreated it progresses rapidly with high mortality. It is considered as a potentially life threatening emergency. We report an acute case of 30 year old female who suffered from cephalalgia which rapidly worsened and ended in her sudden and unexpected death. Magnetic resonance imaging (MRI) of the brain was obtained. Features demonstrated on MRI scan were consistent with a diagnosis of intraventricular NCC.

Copy Right, IJAR, 2018,. All rights reserved.

\section{Introduction:-}

Neurocysticercosis is the most important helminthic central nervous system (CNS) infection worldwide and increasing travel and migration has led to a growing number of patients being diagnosed in non-endemic countries ${ }^{1}$.

The majority of the published literature relates to the parenchymal form of the disease, and there is a paucity of epidemiological studies and high quality evidence to guide the management of the intraventricular variant. It is caused by the larval stage of the tapeworm Taeniasolium, ingested by humans who become dead end intermediate hosts. ${ }^{2}$

The clinical manifestations of the disease vary with the size and location of cysticerci and the intensity of the host's immune response.

Oncospheres migrate to various tissues, most commonly the CNS, eyes, muscles and subcutaneous tissues, and once fully developed as viable cysts, go through three consecutive distinct stages in the process of degeneration: colloidal, granular and calcified forms. ${ }^{3}$ In intraventricularneurocysticercosis, the larvae reach the cerebral ventricles via the choroid plexus and cause symptoms either from obstructing CSF flow, ependymitis or both. ${ }^{4,5}$

Intraventricular disease has been reported to occur in between $7.3 \%$ and $61.3 \%$ of cases of neurocysticercosis. ${ }^{6}$ Cysts are most often found in the fourth ventricle (range 43\%-70\%) followed by the lateral (11\%-43\%) and third $(1 \%-29 \%)$ ventricles with a minority in the Sylvean aqueduct $(7 \%-9 \%)^{7,8}$ Compared to parenchymal 
neurocysticercosis, patients with intraventricular disease have a worse overall outcome and most deaths occur in this subgroup. 9

\section{Case Report:-}

30 year old female presented with the history headache from last 3 weeks. The headache became worse over the last 4-5 days and was associated with vomiting. One day before she had high grade fever and she lapsed into altered sensorium. There was no history of seizure, trauma, blurring of vision and photophobia and no other relevant fever history. Patient had previously been healthy without medical concerns. Her family denied previous history of consuming raw pork meat.

At the time of presentation patient was in altered sensorium (GCS-E1V1M2) and pupils were bilaterally constricted and reacting to light. She had fever of 101 degree F on examination she had neck stiffness with no focal neurological deficits. No pathological reflex was detected. She had leucocytosis of 21500 of which $83 \%$ were neutrophills and blood test including HIV serology, thyroid function test, viral markers, coagulation profile, vitamin B12 levels, infectious profile - IgMtyphi dot, malaria Ag, urine culture and blood culture was unremarkable. However plasma procalcitonin levels was $30.80 \mathrm{ng} / \mathrm{ml}$ and lactate levels was $11.06 \mathrm{mmol} / \mathrm{L}$. NCCT Brain showed non communicating hydrocephalus with normal sized 4th ventricle. Empirical treatment for bacterial meningitis was started and patient was intubated in view of poor GCS and was put on ventilator. Guarded lumbar puncture was done and CSF collected contained all 35-40 mononuclear cells per mm3 and no organisms were identified on gram stain or standard bacterial culture. Also, CSF ADA levels were normal. The CSF levels of glucose and protein were $8.4 \mathrm{mg} / \mathrm{dl}$ and $239 \mathrm{mg} / \mathrm{dl}$ respectively. Patient's general condition deteriorated. Subsequent MRI brain with contrast showed an intraventricular non enhancing cystic lesion in the third ventricle causing mild obstructive hydrocephalus with possibility of intraventricular NCC (Figure 1). She was immediately treated with intravenous dexamethasone and praziquantel therapy. Emergent extra ventricular drain was put. The patient did not improve as the hydrocephalus did not resolve due to intraventricular inflammation presumably caused by the toxic effect of the cyst fluid. 2 days later, she suffered sudden cardiac arrest and despite resuscitation she died.

\section{Discussion:-}

NCC is considered to be the most common parasite infection of the central nervous system. Although being considered eradicable it is still largely endemic in countries and regions where pigs are raised in primitive conditions and where pork is consumed raw or undercooked. Acute symptomatic seizures are the most common manifestations. The other clinical conditions include headache, hydrocephalus, chronic meningitis, focal neurological deficits, and psychological disorders. ${ }^{10}$ Altered sensorium and raised intracranial pressure (ICP) may require ventilatory support in an intensive care unit.

Intraventricular NCC is a less common appearance of NCC; but is potentially serious and mobile and can steadily increase in volume thereby blocking the CSF flow causing obstructive hydrocephalus. Persistent or intermittent symptoms of raised intracranial pressure are often the first clinical signs of this presentation but sudden death has also been reported. Therefore, immediate diagnosis and surgical intervention are imperative. The endoscopic excision of the cyst is currently the method of choice since it is minimally invasive technique with good outcomes. In cases where hydrocephalus persists after cyst removal, ventriculo peritoneal shunting is necessitated, although outcomes are less favorable.

MRI is considered the best neuroimaging tool for the detection of degenerating and innocuous cysticerci, while CT is better for calcified lesions. Control of $\mathrm{ICH}$, neuroendoscopic removal of cysts from ventricles, judicious use of antiparasitic drugs with steroids are the mainstays of therapy.

\section{Conclusion:-}

Intraventricular disease occurs in a significant minority of patients with neurocysticercosis, which is no longer an infection that is restricted geographically to resource-poor regions. The presentation is with features of raised intracranial pressure and the untreated the mortality is high. The diagnosis is based on imaging with MRI, but serological tests, PCR, histopathology and direct surgical visualisation are important supplementary diagnostic tools. Treatment is mainly surgical, preferably using a neuroendoscopic technique, but patients should receive antihelmintic treatment with concomitant corticosteroids to reduce incidence of shunt failure if a ventricular shunt is inserted and to treat undiagnosed viable lesions elsewhere. 


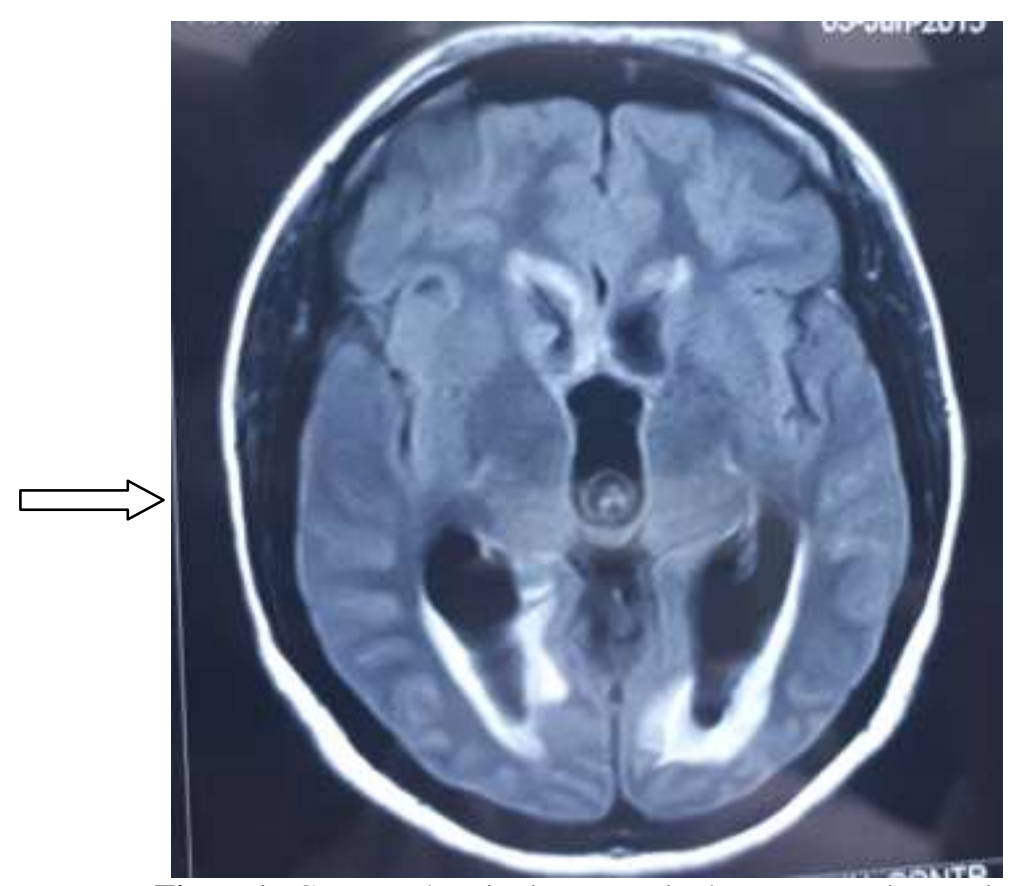

Figure1:-CEMRI showingintraventricularNeurocysticercosis.

\section{References:-}

1. Ndimubanzi PC, Carabin H, Budke CM, Nguyen H, Qian YJ, Rainwater E, et al. A systematic review of the frequency of neurocysticercosis with a focus on people with epilepsy. PLoSNegl Trop Dis 2010; 4: e870.

2. Prasad KN, Prasad A, Verma A, Singh AK. Human cysticercosis and Indian scenario: A review. J Biosci 2008;33:571-82.

3. Garcia HH, Del Brutto OH. Neurocysticercosis: updated concepts about an old disease. Lancet Neurol 2005; 4: 653-661.

4. Cuetter AC, Andrews RJ. Intraventricularneurocysticercosis: 18 consecutive patients and review of the literature. Neurosurg Focus 2002; 12: e5.

5. Sinha S, Sharma BS. Intraventricularneurocysticercosis: a review of current status and management issues. Br J Neurosurg 2011; 26: 305-309.

6. Cuetter AC, Garcia-Bobadilla J, Guerra LG, Martinez FM, Kaim B. Neurocysticercosis: focus on intraventricular disease. Clin Infect Dis 1997; 24: 157-164

7. Goel RK, Ahmad FU, Vellimana AK, Suri A, Chandra PS, Kumar R, et al. Endoscopic management of intraventricularneurocysticercosis. J ClinNeurosci 2008; 15: 1096-1101.

8. Husain M, Jha DK, Rastogi M, Husain N, Gupta RK. Neuroendoscopic management of intraventricularneurocysticercosis (NCC). ActaNeurochir 2007; 149: 341-346.

9. DeGiorgio CM, Houston I, Oviedo S, Sorvillo F. Deaths associated with cysticercosis. Report of three cases and review of the literature. Neurosurg Focus 2002; 12: e2.

10. Garcia HH, Gonzalez AE, Evans CA, Gilman RH. Cysticerosis Working Group in Peru. Taeniasoliumcysticercosis. Lancet 2003;362:547-56. 